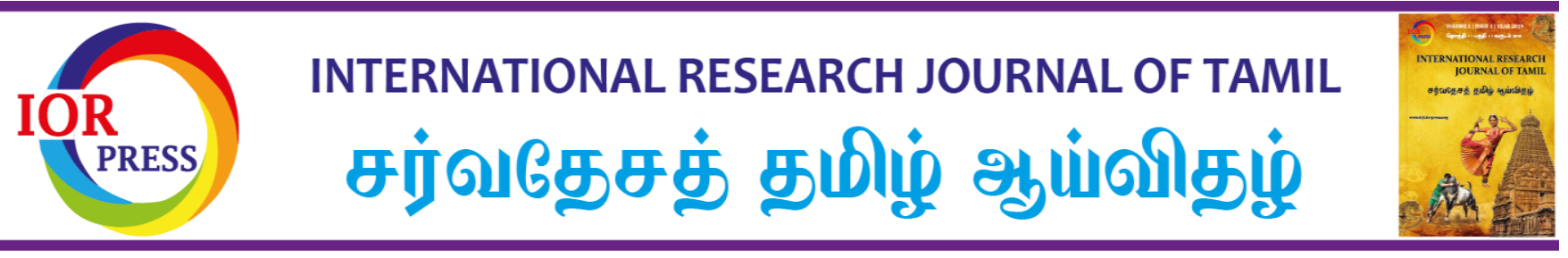
OPEN ACCESS

என்கதை நூல் வழி நாமக்கல் கவிஞர் இராமலிங்கம்
படைப்பும் வாழ்வும்

மா. தேவி அ, *, சு. பாலசுப்பிரமணியன் அ

அ தமிழியல் மற்றும் பண்பாட்டுப்புலம், தமிழ்நாடு திறந்தநிலைப் பல்கலைக்கழகம், சென்னை-600015, தமிழ்நாடு, இந்தியா.

\title{
The work and life of Namakkal Kavignar Ramalingam through my story book
}

\author{
M. Devi ${ }^{a}{ }^{*}$, S. Balasubramaniyan ${ }^{b}$ \\ a School of Tamil and Cultural Studies, Tamil Nadu Open University, Chennai-600015, Tamil Nadu, India.
}

* Corresponding Author: desavi56@gmail.com

Received: 03-05-2021 Revised: 06-09-2021 Accepted: $07-09-2021$

Published: 11-09-2021

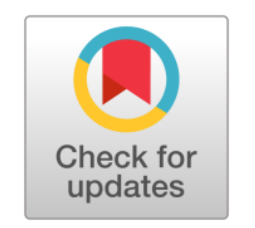

\begin{abstract}
Various types of literature in Tamil have appeared and developed over time. Literature refines our lives. The literature that appeared in each period is able to reveal the living environment of the people of the respective period. Sangam Literature, Sangam Forgotten Literature, Devotional Literature, Folk Literature, Short Story, Novel, Renewal Poetry, Drama, Prose Literature. In this order his historical literature appears and develops. Her history is a collection of events that took place in a person's life. Traces of his historical literature can be found in the Sangam literature. When he and his friend Kopperuncholan, who was dying in the north, went to die in the north, many witnesses there asked why he had not lost his hair for so long. He has the best character wife in life, and people. He says that the Evelars who do not say what he thinks, and that the king is a good protector. And in our town live many learned, virtuous, well-meaning people with goals and principles. So I don't care. So he says I don't have gray hair. Through this, the news about Pichirantaiyar, his hometown, the witnesses in Avur, the people, the king and the evildoer are revealed. And he records through his songs that he lived a quiet contented life without any problems or interruptions. The above biographical notes are able to know the capital of his historical literature. Autobiography is written by a wide variety of writers, political leaders, scholars, and writers from all walks of life. One of the most significant of these biographies is considered to be that of the poet Ramalingam Pillai. The poet Ramalingam of this book is not only talking about the child's own life. Rather it speaks to the community as well. Because the poet Ramalingam Pillai has expressed in his works that he loved this community and what he experienced in his life. In particular, many of the events under the headings of Prayer, Thirukkural Pride, Gandhi, Nattukkummi, Feminism, Bharathidarshanam can be traced back to his works.
\end{abstract}

Keywords: Reflection, Gandhian Thought, Patriotism, Writing, Liberation.

\section{முன்னுரை}

“வையத்து வாழ்வாங்கு வாழ்பவன் வானுறையும்

தெய்வத்துள் வைக்கப் படும்"

என்பது வள்ளுவனின் வாக்கு ஆகும் (Ramalingam Pillai, 1957). பெருமை, புகழ், சிறப்பு, வியக்கத்தக்க செயல்கள் செய்து வாழ்ந்த சான்றோர் பெருமக்கள் வரலாற்றில் பேசப்படுகின்றனர். தன்வரலாறு என்பது 
ஒரு மனிதனின் பிறப்பு முதல் இறப்பு வரை உள்ள வாழ்க்கை நிகழ்வுகளை உண்மைத் தன்மையுடன் முறைப்படுத்திக் கூறுவது ஆகும். அவ்வகையில் பல்வேறு சான்றோர்கள் தன்வரலாற்று இலக்கியங்களை படைத்து உள்ளனர். நாமக்கல் கவிஞர் இராமலிங்கம் பிள்ளை அவர்கள் என்கதை (சுயசரிதை) எனும் அவரது சுயசரிதை நூலில் கூறப்பட்டுள்ள கருத்துகள், செய்திகள், எண்ணங்கள் போன்றவை அவரது படைப்புகளில் பிரதிபலித்திருக்கின்றனவா என ஆய்வதே இக்கட்டுரையின் நோக்கமாகும் (Ramalingam Pillai, 2006).

\section{பிரார்த்தனை:}

நாமக்கல் கவிஞர் இராமலிங்கம் சேலம் ஜில்லா (1944) போர்டுவில் ஒரு உறுப்பினராக இருந்தார். அப்போது இராமலிங்கம் அவர்களின் பாடல்களை அச்சிட்டு எல்லா பள்ளிக்கூடங்களிலும் வழங்க தீர்மானம் நிறைவேற்றப்பட்டது.

"அதற்கிணங்கி நான் சில பாட்டுக்களை சேர்த்து அதற்குப் பிரார்த்தனை என்று பெயரிட்டு என் சொந்த முயற்சியாகவே அச்சிட்டு போர்டாருடைய அங்கீகாரத்துக்கு அனுப்பினேன்"

இவ்வாறு மாணவர்கள் தினந்தினம் பாடும் வகையில் நாட்டுப்பற்று, மொழிப்பற்று, தன்னம்பிக்கை உள்ள பாடல்களை எழுதி தொகுத்துள்ளார் (Ramalingam Pillai, 2006). அதில்

“உலகெலாம் காக்கும் ஒருதனிப் பொருளே

உன்னருள் நோக்கி இன்னுமிங் குள்ளோம்

சொந்த நாட்டினில் தோன்றிடும் செல்வம்

எந்த நாட்டிலோ எங்கோ போய்விடக்

கஞ்சியு மின்றிக் கந்தையு இன்றி

அஞ்சிய வாழ்வின் அடிமையிற் கிடந்து

மங்களம் பாடி மகிழ்ந்திடத் தருவாய்

எங்கும் இருக்கும் எழிலுடைச் சோதி"

-நா. க. பாடல் - தொ. 1.

எனும் வரிகள் கவிஞரின் தேசப் பற்றினை உணர்த்துவதோடு, மாணவர்களும் சிறந்த தேசப்பற்றாளர்களாக உருவாக வேண்டும் என்பதை இந்த பாடல் கூறுகின்றன (இராமலிங்கம் பிள்ளை, 2001). அதாவது, இந்திய நாடு இந்தியர்களுக்கு இல்லையோ எனவும், காடு, மலை, நதி, கனி இங்கு பிறந்த எங்களுக்கு இல்லையோ, சொந்த நாட்டில் தோன்றிய செல்வம் வெளியில் செல்வதையும், கொள்ளை நோயினால், கந்தை இன்றி அஞ்சி வாழ்கின்றோம் எனக் கூறுகிறார். ஆண்மை, அறிவு, அன்பு ஆற்றல், சோறு, துணி, குடிமை, நீதி கொடுத்து எங்களின் வாழ்க்கையை செம்மையுறுமாறு வேண்டுகிறார். இதனை நாமக்கல் கவிஞர் பாடல்களில் பிரார்த்தனை என்னும் தலைப்பின் கீழ் அமைந்துள்ளதை அறிய முடிகிறது.

\section{திருக்குறளின் பெருமை:}

திருக்குறளின் சிறப்பானது நாம் அனைவரும் அறிந்த ஒன்றாகும். இராமலிங்கனாருக்கு சிறு வயதிலேருந்து திருக்குறள் மீது ஈடுபாடு அதிகம் கொண்டவர் எனக் கூறலாம். ஏனென்றால் திருக்குறள் ஒரு குறுகிய சொற்றொடர் - படிப்பதற்கு எளிமையானவை, ஆழம் நிறைந்த கருத்துகளை கொண்டவை. மேலும் திருக்குறளைப் படிக்க விரும்பினால் முறையாக ஆசானிடம் சென்று தான் கற்க வேண்டும். ஏனெனில் திருக்குறள் பரிமேலழகர் உரை சற்று கடினமானதாகவும், யாரேனும் ஒருவர் பொருள் விளக்கும்படி, விளக்கிக்கூறினால் மட்டுமே கருத்தை உணரமுடியும் என்ற கருத்து அக்காலத்தில் 
பரவலாக இருந்து வந்தது. கவிஞர் சிறையில் இருக்கும் போது பரிமேலழகர் உரை கடினமானதாக இருப்பதாகவும், தாங்கள் உரை எழுத வேண்டுமெனவும்,

“புரியாத இடங்களை விட்டுவிட்டுப் புரியக்கூடிய குறள்களுக்காவது எல்லாரும் படித்தறியக்கூடிய எளிய நடையில் ஒரு உரை எழுதி வெளியிடும் வேலையை நீங்கள் செய்தே ஆக வேண்டும். எழுதிக் கொடுங்கள் நான் அச்சிட்டு வெளியிடுகிறேன்" - (Ramalingam Pillai, 2006).

என்று அவரது நண்பர்கள் கூறி வலியுறுத்தியதை தனது சுயசரிதையில் பதிவு செய்கிறார்.

“அது நாள் வரையிலும் தான் கற்றதும் கேட்டதுமாகிய திருக்குறள் உரைகளையெல்லாம் கட்டிச் சுருட்டி என் மனதின் ஒருபுறத்தில் ஒதுக்கி வைத்து விட்டு, அகராதியும் அறிவும் அனுபவமும் ஆகிய இவை மூன்றையும் துணையாகக் கொண்டு மிகவும் துணிச்சலோடு எனக்குத் தோன்றிய உரைகளை எழுதினேன்" - (என்கதை - 274,2006)

திருக்குறளுக்கு பரிமேலழகர் உரை கடினமாக உள்ளது என பலரும் வேண்ட அவரே உரை எழுதத் தொடங்கினார். அவர் எழுதிய உரையை தொகுத்து ‘திருக்குறள் புது உரை’ என்னும் நூலாக வெளியிட்டுள்ளார் (இராமலிங்கம் பிள்ளை, 2001; இராமலிங்கம் பிள்ளை, 1957). அதில் பரிமேலழகர் உரையிலிருந்து தனது உரையை எளிமைப்படுத்தியுள்ளார் என்பதையும், சுயசரிதை நூலுக்கு சான்றாக

"உண்ணாமை யுள்ள துயிர்நிலை ஊனுண்ண

வண்ணாத்தல் செய்யா தளறு" (Ramalingam Pillai, 1957).

\section{பரிமேலழகர் உரை}

உயிர்நிலை ஊன் உண்ணாமை உள்ளது - ஒரு சார் உயிர் உடம்பின் கண்ணே நிற்றல் ஊனுண்ணாமை என்கின்ற அறத்தின் கண்ணது, உண்ண அளறு அண்ணாத்தல் செய்யாது - ஆகலான் அந்நிலைகுலைய ஒருவன் அதனை யுண்ணுமாயின், அவனை விழுங்கிய நிரயம் பின் உமிழ்வதற்கு அங்காவது.

உண்ணப்படும் விலங்குகள் அதனால் தேய்ந்து நிலவாக, ஏனைய பலவாய் வருதலின் உண்ணாமை உள்ளது உயிர்நிலை என்றார். 'உண்ணின் என்பது உண்ண எனத்திரிந்து நின்றது. ஊன் உண்டவன்அப்பாவத்தான் நெடுங்காலம் நிரயத்துள் அழுந்தும் என்பதாகும். கொலைப்பாவம் கென்றார் மேற்றலின் மின ஊன் உண்பார்க்கும் பாவம் இல்லை என்பாரை மறுத்து, அஃது உண்டு என்பது இவ்விரண்டு பாட்டாலும் கூறப்பட்டது.

\section{இராமலிங்கனார் உரை}

மனிதனுடைய உயிர் வாழ்க்கைக்கு வேண்டிய எல்லா சத்துப்பொருள்களும் புலால் உண்ணாமலேயே கிடைக்கின்றன. கொடிய இடமென்றும் பாவிகளை பழிவாங்கும் வெகு பயங்கரமான இடமென்றும், சொல்லப்படுகின்ற நரகமும் கூட உயிர்போன உடலை உள்ளே விடுவதில்லை. ஒருவன் நரகத்துக்கு போனான் என்றால் அவனுடைய உயிர்தான் நரகத்தில் தள்ளப்படுவதாக சொல்லப்படுகிறதே தவிர, உயிர் போன அவனுடைய பிணம் அங்கே அனுமதிக்கப்படுவதில்லை. ஆதலால் நரகமும் கூட உட்கொள்ள மறுத்துவிடுகின்ற பிணத்தை மனிதன் உட்கொள்ளுவதேன்? ஊனுண்பது நரகமும் மறுக்கின்ற செய்கையானதால் சொர்க்கத்தைப்பற்றி நினைக்கவும் இடமில்லை. அதனால் மறுமைப்பயனும் நிச்சயம் இல்லை. பின் ஏன் உண்பதற்காக பிற உயிரைக் கொல்ல வேண்டும் என தெளிவாக எளிமையாக உரை எழுதியுள்ளார். இவ்வாறு அவர் என்கதையில் குறிப்பிட்டபடி திருக்குறளுக்கு உரை எழுதியுள்ளார் என்பது தெரிய வருகிறது. சொல் மட்டும் அல்ல செயலும் செய்து காட்டியவர் 


\section{காந்தி}

1966-ம் ஆண்டு ஏற்பட்ட வங்காள பிரிவினையால் ஏற்பட்ட தேசிய கிளர்ச்சியே இராமலிங்கனாரை அரசியலில் ஈடுபட வைத்தது. அப்போது தென்னாப்பிரிக்காவை விட்டு காந்தியடிகள் இந்தியாவிற்கு வந்து இந்திய சுதந்திர போராட்டத்தில் தலைவராக ஆரம்பித்து பணிகளைத் தொடங்கினார். அப்போது 'ஹிந்து சர்வ கலாசாலை'யின் திறப்பு விழாவிற்கு காந்தியடிகள் வருகை புரிந்திருந்தார். அவரை பேசும்படி அழைப்பு விடுவிக்கப்பட்டது. கோடானு கோடி மக்கள் உண்ணக் கஞ்சியும் உடுக்கக் கந்தையும் இல்லாமல் தவிக்கும் இந்த நாட்டில், இன்னும் உங்களில் பல கோடிக்கணக்கான ஆபரணங்களை அணிந்து, ஆடம்பரத்தில் வாழ்வது பாவமல்லவா? எனப் பேசினர். இதைக்கேட்ட இராமலிங்கனாருக்கு உள்ளத்தில் உணர்ச்சி ஏற்பட்டது. காந்தியடிகள் மகா புருஷன் என எண்ணி பெருமிதம் அடைந்தார்.

அவருடைய உணர்வுப் பூர்வமான பேச்சைக் கேட்டு மனதில் பெரும் எழுச்சியும், மரியாதையும் தோன்றியது.

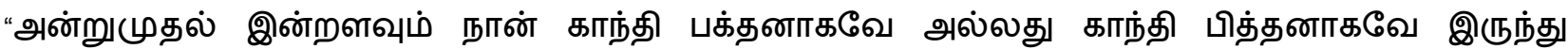
வருகின்றேன். கல்கத்தா பயணத்துக்குப்பின் என் வாழ்க்கை முற்றிலும் மாறி விட்டது. காந்தியம் ஒன்றே நம் நாட்டின் விடுதலைக்கு மட்டுமல்லாமல் உலக நலத்துக்கும் கதி மோஷம் என்று கருதலானேன்" (Ramalingam Pillai, 2006)

இவ்வாறு அவர் காந்திக் கொள்கையின் மீது ஈடுபாடுகொண்டு பக்தனாகவும், பித்தனாகவும், காந்திய வழியில் வாழவேண்டும் என விரும்பினார். இதனை வெளிப்படுத்தும் வகையில் தனது படைப்புகளில் காந்தியைப் பற்றி பாடியுள்ளார் (Ramalingam Pillai, 2006).

“கல்லாலும் செம்பாலும் கடவு ளாக்கிக்

கற்பூரம் காட்டிவிட்டால் போதும் என்றே

எல்லாரும் நினைத்துவிடச் செய்து நித்தம்

தெய்வத்தை ஏமாற்றி வாழ்ந்தோம் என்று

சொல்லாலும் செயலாலும் எண்ணத் தாலும்

அர்த்தமுள்ள பக்தினெறி சொல்லும் தந்து

கல்லாத எளியவர்க்கும் கடவுள் தன்மை

கண்ணார காட்மெங்கள் காந்தி வாழ்க்கை"

என்ற வரிகள் காந்தியடிகள் வழி வாழ வேண்டும் என்று இராமலிங்கம் கூறியுள்ளதாக விளக்குகிறது (இராமலிங்கம் பிள்ளை, 2001). மேலும் மகாத்மா, கண்கண்ட தெய்வம், கடவுளைக்காட்டும் காந்தி, அன்பின் உருவம், இணையிலா காந்தி, அற்புதன் காந்தி, சத்தியமூர்த்தி நம் காந்தி, எச்சரிக்கை, காந்தி வழி, காந்தியமும் தமிழும், காந்தியச்சேவை என்னும் பல்வேறு தலைப்பின்கீழ் காந்தியின் அஹிம்சை, தொண்டு, தாய்மொழிப்பற்று, கொள்கைகள், சேவை, கருணை போன்றவற்றை தம் படைப்பகளில் வெளிப்படுத்தியுள்ளதன் மூலம் தனது சுயசரிதையில் குறிப்பிட்டுள்ளபடி தம் படைப்புகளிலும் காந்திய சிந்தனைகளை மக்களுக்கும் கொண்டு சேர்த்திருக்கிறார்.

\section{நாட்டுக் கும்மி}

இராமலிங்கனார் தனது நண்பர்களுடன் அமர்ந்து உரையாடிக் கொண்டிருக்கும் போது பாடுமாறு கூறினார்கள்.

“என் தமிழறிவையும் கவித்துவத்தையும் அறிந்து ஆனந்தமடைந்து ஆசிர்வதித்தார். அதற்கு ஏழு வருஷத்துக்கு முன்னால் பாடிய என் நாட்டுக்கும்மியை நான் பாடிக்காட்டினேன். அதைக் கேட்ட பிள்ளையவர்கள் சிறு பையனைப் போலத் துள்ளிச் சிரித்து பரவசப்பட்டு என்னைப்பல விதமாகப் பாராட்டினார்கள்" (Ramalingam Pillai, 2006) 
என அவர்பாடிய நிகழ்வு குறித்து என்கதையில் குறிப்பிடுகிறார் (இராமலிங்கம் பிள்ளை, 2001). நாட்டுக்கும்மி சிறுகாப்பியம் போன்றது. இந்திய நாட்டைப் பற்றியது. தமிழ் மக்களிடை யே இன்றும் மிகவும் செல்வாக்குடன் விளங்குகின்ற 'கும்மி' என்னும் நாட்டுப்பாடல் வகையில் புனையப் பெற்றுள்ளது. இப்பாடல்களைப் பெரும்பாலும் பெண்களே வட்டமாக நின்று பாடிக்கொண்டே இரு கைகளையோ அல்லது கைகளிலுள்ள கோல்களையோ ஒன்றோடொன்று தாளத்திற்கு ஏற்ப அடித்து ஓசை எழுப்பி சுற்றி ஆடுவர். இராமலிங்கனாரின் மனவெழுச்சியைக்

"கும்மியடி பெண்கள் கும்மியடி யுங்கள்

கொத்துச் சரப்பனி சத்தமிட

நம்மையெ லாம் பெற்ற இந்திய நாட்டின்

நன்மையைக் கோரி யடியுங்கடி".

\section{தற்கால நிலைமை}

“கிட்டுங்க டிமலர் கொட்டுங்க டிகையைத்

தட்டுங்கடி கண்ணீர் சொட்டுங்கடி

மட்டில் புகழ்கொண்ட இந்திய தேவியின்

மாட்சிமை கெட்டதைக் கேளுங்கடி

கெட்டோம டிகுடி கெட்டோம டிநாம்

பட்டோம டிவெகு கஷ்டமடி

முட்டத் தரித்திரம் கிட்டி முளைக்குது

மூதேவி நம்மை முறைமை கொண்டாள்"

என இந்திய நாட்டில் சொந்த மக்களே புறக்கணிக்கப்படுவதை ஒரு தாய்க்கு ஒப்பிடுவதைப் பார்க்க முடிகிறது (Ramalingam Pillai, 2001). அத்தாயின் முன்னைய புகழோங்கிய நிலையையும், அந்நிய வெள்ளை மாது ஒரருத்தியின் செல்வாக்குக்குட்பட்டு இந்திய மக்களால் புறக்கணிக்கப்பட்டதையும் அவளுக்கு ஏற்பட்ட பரிதாப நிலையையும் கூறுகிறார். மேலும் தங்களிடையே காணப்படும் வேற்றுமைகளை எல்லாம் மறந்து, ஒற்றுமையாக இருந்து இந்திய தாயை வணங்குமாறும், சுதேசியத்தைப் பேணுமாறும் தமது சுயசரிதையில் கூறியது போலவே படைப்புகளில் வெளிப்படுத்தியதைக் காணமுடிகிறது.

\section{பெண்மை}

இராமலிங்கனார் இல்லற வாழ்வில் பற்றற்றவராகவே இருந்தார். தனது தந்தையின் கட்டாயத்தின் பேரில் நெருங்கிய உறவினரின் மகளான முத்தம்மாளை திருமணம் செய்து கொண்டார். இவர் தனது மனைவியிடம் அன்பு காட்டாமல் அருவருப்பாக நடந்து கொண்டார். ஆனால் முத்தம்மாளோ ‘கல்லானாலும் கணவன் புல்ானாலும் புருஷன், கணவனே கண்கண்ட தெய்வம் என்ற மனப்பான்மையில் தனது வழக்கமான பணிவிடைகள் செய்து வந்தார். காலங்கள் பல கடந்தது. ஒரு நாள் முத்தம்மாள், இராமலிங்கனாரிடம் அத்தான் பேசேமாட்டீர்களா நான் என்ன தப்பு செய்தேன் என்று கேட்டாள். நீ வேண்டுமானால் உங்கள் வட்டுக்கு போ என்றார். அதைக்கேட்டு பரவாயில்லை நீங்கள் என்னிடம் பேசவேணாம் நான் இங்கேயே இருந்து உங்களைப் பார்த்துக் கொள்கிறேன். அப்போது பதில் கூறமுடியாமல் தவித்தார். அழுதழுது கண்கள் இரண்டும் குளம் போல தேங்கியிருந்தன.

“எனக்கு முன்னால் அடிபணிந்து அபயங்கோரிய முத்தம்மாளின் முகத்தில் ‘பெண்மை’என்னும் பெரும் பொருளைக் கண்டேன். அகழ்வாரைத் தாங்கும் நிலம் போலத் தம்மை இகழ்வார்ப் பொறுத்தல் தலை என்ற ஆபூர்வக் குணத்துக்கும் அதிகப்பட்டதென்று சொல்லும்படி தன்னை இகழ்ந்தவனைச் 
சரணடைந்த அவளுடைய பெருந்தன்மையும் அப்படிப்பட்ட ஒரு பெண் ரத்தினத்தை அது நாள் வரயிலும் அவமதித்து நடந்து கொண்ட என் அற்பத்தனமும் எனக்குப் புலனாயின" (Ramalingam Pillai, 2006)

என தனது வாழ்க்கையில் நடந்தவற்றை சுயசரிதையில் கூறியுள்ளார் (Ramalingam Pillai, 2006)

மேலும் தனது வாழ்வில் நடந்த காட்சியை அவரது படைப்பிலும் வெளிப்படுத்த எண்ணி "பெண்மை" என்னும் தலைப்பின் கீழ் ஒரு பெண் வகிக்கும் பதவிகளை

"அன்பும் ஆர்வமும் அடக்கமும் சேர்ந்தும்

வஞ்சனை என்பர் வஞ்சகமுடையோர்"

தாய், தாரம், உடன்பிறப்பு, மகள், சேவை செய்பவள், அன்பு காட்டுபவள் என ஒரு பெண் இச்சமுதாயத்தில் எவ்வாறெல்லாம் வேறுபட்டு இருக்கிறாள் என்பதை கவிஞர் வெளிப்படுத்தியதை அறிய முடிகிறது (Ramalingam Pillai, 2001).

\section{பாரதி தரிசனம்}

இந்தியா நமக்குச் சொந்தமான நாடு. அதை இந்தியரல்லாதவர்கள் ஆண்டு வருகின்றனர் என்ற சுதந்திர உணர்ச்சி பள்ளிக்கூடங்களில் பரவ ஆரம்பித்த காலம். பாரதியாருடைய சுதந்திர வெறி, கவிதை, பிரசங்கங்களில் கேட்கும் போது இராமலிங்கரின் உடலும், உள்ளமும் துடிக்கும். பாரதியார் சென்னையிலுள்ள மூர்மார்க்கெட்டில் மாலைநேரத்தில் பிரசங்கம் செய்வார். ஒரு நாள் கூட்டத்திற்கு இடையில் “என்று தணியுமிந்த சுதந்திரத் தாகம்" என்ற பாட்டை பாடி ஆவேசமாக பிரசங்கம் செய்து கொண்டிருந்தார். அதைக் கேட்டுக்கொண்டிருந்த இராமலிங்கனாருக்கும் பாரதியை பார்க்க வேண்டும் என்ற அவா எழுந்து, பின்பு அவரைப் பற்றிய பேச்சுகள் எழும் போதெல்லாம் எப்படியாவது பாரதியின் பாட்டை நேரடியாக ஒருமுறையாவது கேட்க வேண்டும் என்ற ஆசையில் இருந்தார். ஒரு நாள் பாரதியார் கானாடு காத்தா என்ற இடத்தில் (காரைக்குடி) வந்திருக்கிறார் என ஒரு செய்தி வந்ததும் அவர் பாரதியாரைப் பார்க்கச் சென்றார்.

அப்போது நண்பர் ஒருவர் பாரதியை அறிமுகம் செய்து வைக்க பிள்ளைவாள் நீர் நம்மை ஓவியத்தில் தீட்டும். நாம் உம்மைக் காவியத்தில் தீட்டுவோம் என்று சொல்லிச் சிரித்தார். பாரதியார் இராமலிங்கத்தை கவிப்பாடுமென்று அழைத்தார். அப்போது

“தம்மராசப் பிறர் ஆள விட்டுவிட்டுத்

தாம் வணங்கி கைகட்டி நின்றபேரும்

இம்மையிலும் மறுமையிலும் இழிவடைந்து

எரிநரகில் அழுந்திடுவார் எந்த நாளும் “15-, (Ramalingam Pillai,2006)

என்ற பாடலைப் பாடியதும் பாரதியார் புகழ்ந்து "பலே பாண்டியா பிள்ளை நீர் ஒரு புலவன், ஐயமில்லை" என மனமார வாழ்த்தினார்.

பாரதியின் மீது கொண்ட அளவுகடந்த பற்றின் காரணமாக தமது படைப்புகளிலும் பாரதியைப் பற்றி பாடியுள்ளார். அவ்வரிகள் வருமாறு

“பெற்றெடுத்த தமிழ்த்தாயைப் பின்னால் தள்ளிப்

பிறமொழிக்குச் சிறப்பளித்த பிழையே நீக்க 
தெற்றென நம் அகக்கண்ணைத் திறந்து விட்ட

தெய்வகவி பாரதி ஆர் ஆசான் திண்ண"

"பாரதி எனும் பெயரைச் சொல்லு - கேட்ட

பயமெனும் பகைவனை வெல்லு.

நேரினி உனக்குநிகர் இல்லை - உடன்

நீங்கும் அடிமைமதனத் தொல்லை"

என் தமிழ்மொழியின் சிறப்பே பாரதி என்றும், பாரதி பெயரைச் சொன்னால் பயம் நீங்கும் எனவும், அவருடைய பாட்டுகளைக் கேட்டால் சோம்பல், மனச்சோர்வு நீங்கி தன்மதிப்பு உயரும் என்றும் தனது படைப்புகளில் வெளிப்படுத்தியுள்ளதை அறிய முடிகிறது.

\section{முடிவுரை}

இராமலிங்கம் பிள்ளை காந்தியின் மீது கொண்ட பற்றின் காரணமாக காந்தி குறித்து பதிவு செய்துள்ளதோடு, காந்தி குறித்து தன் வரலாற்று நூலிலும், படைப்புகளிலும் வெளிப்படுத்தியுள்ளார் என்பதை அறிய முடிகிறது. பெண்களின் பெருமையை தமதும னைவி மூலம் உணர்ந்த, இராமலிங்கனார் பெண்களின் பெருமையை பிறர் அறியும் பொருட்டு தம் படைப்புகளிலும் படைத்துள்ளார் என்பது புலப்படுகிறது. இராமலிங்கனார் பாரதியை நேரில் கண்டு அதாவது தரிசித்ததைத் தமது படைப்புகளில் பாரதியின் ஆற்றல், தேசபக்தி, சுதந்திர உணர்ச்சி என்ற பல்வேறு கருத்துகளாகப் படைத்து, இந்தச் சமூகத்திற்கு அளித்துள்ளார் என்பது தெரிய வருகிறது. மேலும் இராமலிங்கனார் தனது சுயசரிதையில் கூறிய கருத்துகளும் தனது படைப்புகளில் வெளிப்படுத்திய கருத்துகளும் வேறு வேறல்ல. தனது வாழ்க்கையில் எப்படி வாழ்ந்தாரோ அந்த வாழ்வையே படைப்பாயிருக்கிறார் கவிஞர். தேசப்பக்தியும், மொழிப்பற்றையும் தனது சிந்தனையாக கொண்டதோடு அதனையே தனது வாழக்கையாகவே மாற்றிக்கொண்டார் எனலாம். அவர் வாழ்ந்தது மட்டுமல்ல தனது எண்ணத்தையும், சிந்தனையும் படைப்புகளின வழி வெளிப்படுத்தி சமுதாயத்தையும் நல்வழிப்படுத்தியுள்ளார். தமிழில் சுயசரிதைகள் இன்று பலரால் எழுதப்பட்டு வருகிறது. ஆனால் இவற்றை விரும்பி வாசிக்கும் நபர்கள் குறைவாகவே உள்ளனர். ஆதலால் இந்நூலில் உள்ள அரிய கருத்துக்கள் பலர் அறியாமலே உள்ளனர். மேலும் இவற்றை கல்லூரி பாடத்திட்டத்தில் சேர்ப்பதின் மூலம் மாணவர்கள் சான்றோர்களின் சுயசரிதையை வாசிப்பாரகள். இதன் மூலம் அவர்கள் தன்னளவிலும், சமுதாயத்திலும் மிகச்சிறந்தவர்களாக வருவார்கள் என கூறலாம்.

\section{References}

Ramalingam Pillai, V., (1957) Thirukkural Puthuurai, Inba Nilayam, Chennai, India.

Ramalingam Pillai, V., (2001) Namakkal Kavignar Ve. Ramalingam Pillai padalgal, Madurai Tamil Literary Compilation Project, Madurai, India.

Ramalingam Pillai, V., (2006) EnKadhai, 1st edition, Sandhya Pathippagam, Chennai, India.

Funding: NIL

Acknowledgement: NIL

Conflict of Interest: NIL

About the License: 


\section{()(1)}

Attribution 4.0 International (CC BY 4.0)
(C) The author 2021. The text of this article is licensed under a Creative Commons Attribution 4.0 International License 